\section{Dr. Marchesoni and Dr. De Lucia reply}

\section{To the Editor:}

We thank Dr. Ozkan and colleagues for their interest ${ }^{1}$ in our article ${ }^{2}$ on how to distinguish psoriatic arthritis (PsA) from fibromyalgia (FM). In our study, as a complement to the clinical findings, we investigated by power Doppler ultrasound (PDUS) the involvement of 10 entheseal sites in a small subgroup of patients selected randomly (30 PsA, $30 \mathrm{FM}$ ). The sites examined were not those included in the Ankylosing Spondylitis Enthesitis Score (MASES), with the exception of the Achilles tendon.

The point of this substudy was to evaluate whether this imaging technique might enhance the distinction between PsA and FM entheseal pain based only on clinical features. However, the reported results should be interpreted with caution for several reasons. First, we reported only a small portion of the data; second, the small number of patients examined did not allow an adequately powered statistical analysis; and third, the objective of the study was not the PDUS evaluation.

Overall, the findings of this substudy showed that PDUS was much more sensitive than clinical examination in revealing entheseal involvement. As PDUS changes may be mechanical or inflammatory, a reliable definition for this distinction would be needed. We used the definition of enthesopathy suggested by the Outcome Measures in Rheumatology Clinical Trials (OMERACT) ${ }^{3}$, which was the most specific one available at the beginning of the study. All the changes indicated by the OMERACT were recorded. Complete data are reported in a supplement of The Journal $^{4}$. As a marker of previous or current inflammation we used hypoechogenicity, bone erosions, and PD signal at bone insertion of the entheses, which sounded reasonable. Inflammatory PDUS changes were found in $70 \%$ of the patients with PsA, in contrast to about $23 \%$ of patients with FM. The number of sites involved and their distributions were other features distinguishing the 2 disorders. More precisely, a receiver-operating characteristic curve analysis of the number of involved entheses revealed that $\geq 3$ involved sites had the best power to discriminate PsA and FM (AUC $0.766,95 \%$ CI $0.695-0.824$; p < 0.001; sensitivity $72 \%$ and specificity $76 \%$ ). The most significant difference between the 2 conditions was seen in the Achilles tendons, where PDUS signs of inflammation were present in 35 and 3 of the 60 tendons examined in PsA and FM, respectively. This result confirms that Achilles tendon involvement is frequent in PsA and specific for this condition.
What we saw in our study was that clinical involvement of this tendon (tenderness at its insertion site) was not more frequent in PsA (about 20\% of the patients) than in FM (about 22\% of patients). This discrepancy between clinical and PDUS findings, which was characteristic of most of the sites examined by PDUS, could be indicative of subclinical enthesitis, but in our opinion it raises the issue of how "enthesitis" should be defined.

In our study, PDUS was much more sensitive than clinical examination in detecting entheseal inflammatory involvement but, as rightly stated by Ozkan and colleagues ${ }^{1}$, "with increased sensitivity comes reduced specificity." In the context of distinguishing between inflammatory and FM entheseal involvement, this implies the risk of overdiagnosing enthesitis in patients with FM. Studies on larger series of patients and with more sophisticated instruments, such as the suggested Madrid Sonographic Enthesis Index ${ }^{5}$, are clearly needed.

ANTONIO MARCHESONI, MD; ORAZIO DE LUCIA, MD, UOC Department of Rheumatology, Istituto Ortopedico G. Pini, Milan, Italy. Address correspondence to Dr. Marchesoni; E-mail: marchesoni@gpini.it

\section{REFERENCES}

1. Ozkan F, Inci M, Ozyurt K, Bakan B. Psoriatic arthritis and sonographic entheseal index [letter]. J Rheumatol 2012;39:2045.

2. Marchesoni A, Atzeni F, Spadaro A, Lubrano E, Provenzano G, Cauli A, et al. Identification of the clinical features distinguishing psoriatic arthritis and fibromyalgia. J Rheumatol 2012;39:849-55.

3. Wakefield RJ, D'Agostino MA, Iagnocco A, Filippucci E, Backhaus M, Scheel AK, et al. The OMERACT Ultrasound Group: Status of current activities and research directions. J Rheumatol 2007;34:848-51.

4. Marchesoni A, De Lucia O, Rotunno L, De Marco G, Manara M. Entheseal power doppler ultrasonography: A comparison of psoriatic arthritis and fibromyalgia. J Rheumatol Suppl. 2012 July;89:29-31.

5. De Miguel E, Cobo T, Muñoz-Fernández S, Naredo E, Usón J, Acebes JC, et al. Validity of enthesis ultrasound assessment in spondyloarthropathy. Ann Rheum Dis 2009;68:169-74.

J Rheumatol 2012;39:10; doi:10.3899/jrheum.120775 